\title{
Effect of external beam radiotherapy on second primary cancer risk after radical prostatectomy
}

Felix Preisser ${ }^{1,2,3}$; Elio Mazzone ${ }^{2,4}$; Sophie Knipper ${ }^{1,2}$; Sebastiano Nazzani ${ }^{2,5}$; Marco Bandini ${ }^{2,4}$; Shahrokh F. Shariat ${ }^{6}$; Michele Marchioni ${ }^{2,7}$; Zhe Tian ${ }^{2}$; Fred Saad ${ }^{2}$; Daniel Taussky ${ }^{8}$; Alberto Briganti ${ }^{4}$; Hartwig Huland ${ }^{1}$; Markus Graefen ${ }^{1}$; Derya Tilki ${ }^{1,9}$; Pierre I. Karakiewicz ${ }^{2}$

${ }^{1}$ Martini-Klinik Prostate Cancer Center, University Hospital Hamburg-Eppendorf, Hamburg, Germany; ${ }^{2}$ Cancer Prognostics and Health Outcomes Unit, Division of Urology, University of Montreal Health Centre, Montreal, QC, Canada; ${ }^{3}$ Department of Urology, University Hospital Frankfurt, Frankfurt, Germany; ${ }^{4}$ Department of Urology and Division of Experimental Oncology, URI, Urological Research Institute, IRCCS San Raffaele Scientific Institute, Milan, Italy; ${ }^{5}$ Academic Department of Urology, IRCCS Policlinico San Donato, University of Milan, Milan, Italy; ${ }^{6}$ Department of Urology, Medical University of Vienna, Vienna, Austria; ${ }^{7}$ Department of Urology, SS Annunziata Hospital, "G.D'Annunzio" University of Chieti, Chieti, Italy; ${ }^{8}$ Department of Radiation Oncology, University of Montreal Health Centre, Montreal, QC, Canada; ${ }^{9}$ Department of Urology, University Hospital Hamburg-Eppendorf, Hamburg, Germany

Cite as: Can Urol Assoc J 2019 November 29; Epub ahead of print. http://dx.doi.org/10.5489/cuaj.6087

Published online November 29, 2019

$* * *$

\section{Abstract}

Introduction: We aimed to investigate the effect of radiotherapy (RT) in contemporary patients treated with radical prostatectomy (RP) compared to RP alone for non-metastatic prostate cancer (PCa) on the incidence of second primary cancers (SPCs).

Methods: Within the Surveillance, Epidemiology, and End Results database (2004-2015), we identified patients with $\mathrm{PCa}$ as the only or first primary cancer, who underwent RP and RT or RP alone. Cumulative incidence plots and multivariable Cox regression models tested for SPC rate differences according to treatment type: RP and RT vs. RP alone. Subgroup analyses focused on pelvic, primary pelvic, and non-pelvic SPCs, as well as on late SPCs ( $>5$ years after PCa diagnosis).

Results: Of 152161 patients, 7.1\% ( $n=10$ 870) received RP and RT. Overall, 6.6 vs. 5.0\% developed SPCs after RP and RT vs. RP alone, respectively ( $\mathrm{p}<0.001)$. Cumulative incidence rates at 10 years after PCa diagnosis for RP and RT vs. RP were 12.0 vs. 8.7\% ( $<<0.001), 2.0$ vs. $1.2 \%(\mathrm{p}<0.001), 2.1$ vs. $1.3 \%(\mathrm{p}<0.001)$, and 9.9 vs. $7.4 \%(\mathrm{p}<0.001)$ for overall SPCs, primary pelvic SPCs, overall pelvic SPCs, and non-pelvic SPCs, respectively. Multivariable Cox regression models revealed an increased risk after RP and RT vs. RP alone for overall (hazard ratio [HR] 1.2; $<<0.001$ ), primary pelvic (HR 1.5; $<<0.01$ ), pelvic (HR1.4; $<0.001$ ), non-pelvic (HR1.1; $<<0.01$ ), late overall (HR 1.2; $=0.01$ ), and late non-pelvic SPCs (HR1.2; $\mathrm{p}=0.03)$. 
Conclusions: RP with RT was associated with moderately increased risk of SPCs compared to RP alone. This observation should be thoroughly discussed at informed consent and considered during followup.

\section{Introduction}

Radiotherapy (RT) combined with radical prostatectomy (RP) may more significantly reduce the risk for biochemical recurrence, clinical progression and survival in patients with adverse pathological characteristics at RP. ${ }^{1-4}$ However, RT may also lead to second primary cancers (SPCs), which may in turn undermine life expectancy. ${ }^{5-7}$ The risk of SPCs might be particularly pertinent in RP candidates, who in general enjoy superior life expectancy to that of the general population. Indeed, the concept of SPC receives an increasingly greater attention in the medical literature. This phenomenon is at least impart related to the increasing cumulative number of prostate cancer $(\mathrm{PCa})$ survivors, who are diagnosed with a SPC. Moreover, the same phenomenon of SPC affects patients with other primaries than PCa. Specifically, SPCs account for about a sixth of all new cancers reported to the National Cancer Institute's Surveillance, Epidemiology and End Results (SEER) Program. ${ }^{5}$

Regarding SPCs related to RT delivered for primary PCa, Abdel-Wahab et al. reported an increased risk for late ( $>5$ years from initial PCa diagnosis) primary pelvic SPCs, within SEER database. ${ }^{8}$ However, they relied on a historic cohort (1973-2002). To address lack of contemporary data, we assessed the effect of RT on the incidence of SPCs in PCa patients treated between 2004 and 2015. The rationale to repeat the analyses stems from the hypothesis that novel RT techniques may contribute to different rates than those reported historically. ${ }^{9}$ To test our hypothesis, we investigated the effect of RT in patients treated with $\mathrm{RP}$ and RT compared to patients treated with RP alone for non-metastatic $\mathrm{PCa}$, on the incidence of overall SPCs, primary pelvic SPCs, overall pelvic SPCs, non-pelvic SPCs, as well as on late SPCs ( $>5$ years from initial PCa diagnosis), within the SEER database.

\section{Methods}

\section{Study population}

Within the SEER database (2004-2015) we identified patients aged $\geq 18$ years, with histologically confirmed adenocarcinoma of the prostate (International Classification of Disease for Oncology [61.9]; histological code: 8140) as their first malignancy (Sequence number $=0$ [one primary only] or 1 [first of two or more malignancies] $){ }^{8,10}$ Exclusion criteria consisted of confirmed metastatic disease at diagnosis (6th and 7th edition of American Joint Committee on Cancer [AJCC] Cancer Staging Manual), unknown metastatic status and unknown prostatic specific antigen value (PSA). Only those patients that underwent RP or a combination of RP and RT were included. Other exclusion criteria consisted of autopsy diagnosed cases or death certificate diagnoses, cases with a follow-up of $<12$ months and those with SPC diagnosis made within 1 year of primary PCa (time to SPC $<12$ months) to eliminate the possibility that PCa was not the first malignancy. ${ }^{8,10}$. D'Amico risk 
stratification was performed, as previously described. ${ }^{11,12}$ These selection criteria yielded 152,161 evaluable patients. This group represented the focus of the current manuscript.

\section{Outcomes}

The overall SPCs were stratified into three regions of origin. First, primary pelvic SPCs were defined as those arising from the bladder, rectum, anus/anal canal/anorectum, or prostate and other malignancies originating from the pelvis (soft tissue, bone and joints, and lymphoma) ${ }^{8,10}$ Second, overall pelvic SPCs included all primary pelvic SPCs in addition to those SPCs arising from the recto-sigmoid region, penis, small intestine (ileum and jejunum), ureter, other urinary and male genital organs, testis, and lymphoma inside the pelvis. ${ }^{8,10}$ Third, all SPCs that did not qualify as either primary pelvic or overall pelvic were defined as nonpelvic SPCs. Finally, all SPCs regardless of their origin were included in the overall SPC category.

\section{Statistical analyses}

Descriptive statistics included frequencies and proportions for categorical variables. Means, medians, and interquartile ranges were reported for continuously coded variables. The Chisquare tested the statistical significance in proportions' differences. The t-test examined the statistical significance of means' differences, respectively.

Cumulative incidence plots were used to graphically depict the incidence of SPCs, according to RP and RT vs. RP only treatment. First, we relied on four separate sets of multivariable Cox regression models that tested the relationship between treatment type (RP and RT vs. RP alone) and SPC risk. The four specific models respectively focused on: 1) overall SPC risk, 2) primary pelvic SPC risk, 3) overall pelvic SPC risk and 4) non-pelvic SPC risk. Subsequently, we repeated the analysis of the four endpoints, namely: overall SPC risk, primary pelvic SPC risk, overall pelvic SPC risk and non-pelvic SPC risk. However, each model only considered late SPCs, those that arose more than five years after PCa diagnosis: 5) late overall SPC risk, 6) late primary pelvic SPC risk, 7) late overall pelvic SPC risk and 8) late non-pelvic SPC risk.

Covariates in all Cox models consisted of age, D'Amico risk stratification, race, marital status and year of diagnosis. R software environment for statistical computing and graphics (version 3.4.0) was used for all statistical analyses. All tests were two sided with a level of significance set at $p<0.05$.

\section{Results}

\section{Study population}

Overall, 152,161 patients with PCa were identified (Table 1). Of those 7.1\% $(n=10,870)$ received RP and RT vs. 92.9\% that underwent RP alone. RP and RT patients were significantly older (median age: 63 vs. 61 years, interquartile range [IQR]: 57-69 vs. 56-66, $\mathrm{p}<0.001$ ), had a higher median prostatic specific antigen value (7.9 vs. $5.6 \mathrm{ng} / \mathrm{ml}, \mathrm{IQR}: 5.2-$ 14.5 vs. $4.4-8.0 \mathrm{ng} / \mathrm{ml}, \mathrm{p}<0.001)$ and more frequently harbored clinical tumor stage $\geq \mathrm{T} 3(9.8$ vs $1.9 \%, \mathrm{p}<0.001)$, as well as Gleason grade group V (19.6 vs. $3.7 \%, \mathrm{p}<0.001)$. Of the 
141,291 patients treated with RP alone, $1.7 \%(n=2,396)$ died due to the SPCs, $1.0 \%(n=1,447)$ due to PCa and $2.3 \%(n=3,275)$ attributed to other causes. Conversely, of the 10,870 patients who received RP and RT, 3.2\% $(n=349)$ died due to SPCs, 6.4\% $(n=695)$ due to PCa and $4.2 \%(n=460)$ attributed to other causes.

\section{Rates of SPCs}

Of all patients, $5.2 \%(n=7,855)$ developed SPCs (Table 2). RP and RT treated patients more frequently developed overall SPCs (6.6 vs. 5.0\%, p <0.001). The median time to SPC was significantly shorter after RP and RT vs. RP (48.4 vs. 51.4months, IQR: 28.2-73.6 vs. 29.278.6 months, $\mathrm{p}<0.001)$. The most frequently recorded SPCs in the overall cohort were those that arose from lung/pleura $(n=1236,0.8 \%)$, skin $(n=914,0.6 \%)$ and bladder $(n=901,0.6 \%)$, in that order. Stratification according to treatment type revealed virtually the same findings for site of origin for RP patients, namely: lung/pleura ( $\mathrm{n}=1102,0.8 \%)$, skin $(\mathrm{n}=851,0.6 \%)$ and bladder $(\mathrm{n}=792,0.6 \%)$, in that order. Conversely in RP and RT patients, bladder cancer represented the second most frequent SPC $(\mathrm{n}=109,1.0 \%)$. Only SPCs arising from lung/pleura $(\mathrm{n}=134,1.2 \%)$ accounted for higher rate and SPC originating from the skin $(\mathrm{n}=63$, $0.6 \%$ ) still represented the third most frequent site.

Stratification of SPCs according to regions of origin, defined as primary pelvic vs. overall pelvic vs. non-pelvic also demonstrated higher rates after RP and RT vs. RP.

Specifically, for the three regions of origin the rates were 1.2 vs. $0.7 \%(p<0.001), 1.3 \mathrm{vs}$. $0.8 \%(\mathrm{p}<0.001)$ and 5.3 vs. $4.3 \%(\mathrm{p}<0.001)$, respectively.

When the region of origin analyses were repeated with the endpoint defined as late SPCs, virtually the same results were recorded. Specifically, for the three regions of origin, late primary pelvic vs. late overall pelvic vs. late non-pelvic, the rates were 0.4 vs. $0.3 \%(p=0.1)$, 0.4 vs. $0.3 \%(p=0.1)$ and 2.2 vs. $1.8 \%(p=0.02)$, after RP and RT vs. RP alone respectively.

\section{Cumulative incidence for SPCs}

In cumulative incidence plots (Figure 1), overall SPC incidence rates at 10 years were $12.0 \%$ for RP and RT vs. $8.7 \%$ for RP alone $(\mathrm{p}<0.001)$ patients (Figure $1 \mathrm{a})$. For the same two groups, at 10 years primary pelvic, overall pelvic and non-pelvic SPC incidence rates were 2.0 vs. $1.2 \%(\mathrm{p}<0.001)$ (Figure $1 \mathrm{c}), 2.1$ vs. $1.3 \%(\mathrm{p}<0.001)$ (Figure $1 \mathrm{~b})$ and 9.9 vs. $7.4 \%$ $(\mathrm{p}<0.001)$ (Figure 1d), respectively.

\section{Multivariable Cox models predicting SPC}

In multivariable Cox models predicting overall SPCs (Table 3), RP and RT represented an independent predictor of overall SPCs (Hazard ratio [HR]: 1.2, 95\%-confidence interval [CI]: $1.1-1.3, \mathrm{p}<0.001$ ), primary pelvic SPCs (HR: 1.5, 95\%-CI: $1.2-1.8, \mathrm{p}<0.01$ ), overall pelvic SPCs (HR: 1.4, 95\%-CI: 1.2-1.7, p<0.001) and non-pelvic SPCs (HR: 1.1, 95\%-CI: 1.05-1.2, $\mathrm{p}<0.01$ ), compared to RP patients. Moreover, in Cox models predicting late SPCs (Table 4), RP and RT also represented an independent predictor of late overall SPCs (HR: 1.2, 95\%-CI: 1.03-1.3, p=0.01) and of late non-pelvic SPCs (HR: 1.2, 95\%-CI: 1.01-1.3, p=0.03), compared to RP patients. However, no association was recorded in models focusing on late primary pelvic SPCs and late overall pelvic SPCs, after multivariable adjustment. 


\section{Discussion}

RT may predispose to SPCs. ${ }^{5,6}$ Previously, Abdel-Wahab et al. reported an increased risk for late ( $>5$ years from initial PCa diagnosis) primary pelvic SPCs, within SEER database. ${ }^{8}$ However, in their report they relied on a historic cohort (1973-2002). Therefore, we assessed the effect of RT on the incidence of SPCs in contemporary PCa patients. The rationale for such initiative is based on novel RT techniques, e.g. image-guided radiotherapy and intensitymodulated radiotherapy. These could have affected SPC rates to a different or possibly lesser extent than historic RT techniques. ${ }^{9}$ To test our hypothesis, we relied on a large contemporary North American population-based cohort and compared the risk of SPCs in patients treated with RP and RP to patients treated with RP alone. Our analyses demonstrated several noteworthy observations.

First, the proportions of patients that developed overall SPCs were 6.6 vs. $5.0 \%$ $(\mathrm{p}<0.001)$ for RP and RT vs. RP, respectively. Moreover, most frequently recorded SPC sites in the overall cohort were those that arose from lung/pleura $(n=1236,0.8 \%)$, skin $(n=914$, $0.6 \%)$ and bladder $(\mathrm{n}=901,0.6 \%)$, in that order. Stratification according to the treatment type revealed the same order for RP treated patients: lung/pleura $(n=1102,0.8 \%)$, skin $(n=851$, $0.6 \%)$ and bladder $(\mathrm{n}=792,0.6 \%)$. Conversely, in RP and RT treated patients, bladder cancer $(\mathrm{n}=109,1.0 \%)$ was the second most frequent SPC site, lung/pleura $(\mathrm{n}=134,1.2 \%)$ ranked first and skin $(n=63,0.6 \%)$ ranked third. Our results corroborate the findings of Abdel-Wahab et al., who also identified lung/pleura cancers as the most frequent SPCs after PCa ${ }^{8}$ Conversely, they identified colon-cancer, as the second most frequent SPC site in the overall cohort and RP treated cohort. This observation may be related to an elevated rate of skin cancers in elderly men that are unrelated to PCa treatment but rather reflect lifestyle. Despite these differences, Abdel-Wahab et al. also identified bladder cancer as second most frequent SPC site in patients treated with RP and RT. This finding is in agreement with our observations regarding the importance of secondary bladder cancer after RP combined with RT. This finding is important in patient counseling, at informed consent and in follow-up planning, when combination of RP and RT is considered.

Second, stratification according to SPC regions of origin (primary pelvic, overall pelvic and non-pelvic) demonstrated higher rates after RP and RT vs. RP. Specifically, the absolute rate differences were 1.2 vs. $0.7 \%(\mathrm{p}<0.001), 1.3$ vs. $0.8 \%(\mathrm{p}<0.001)$ and 5.3 vs. $4.3 \%(p<0.001)$, respectively for the three regions of origin. Finally, stratification according to regions of origin in late SPCs showed higher SPCs rates for RP and RT in the non-pelvic SPCs $(2.2$ vs. $1.8 \%, \mathrm{p}=0.02)$. This region-specific information confirms the overall rates and demonstrates the generalizability of our findings to all three specific anatomic regions. Unfortunately, as outlined in the limitations section, sample size limitations regarding the number of patients at risk and the numbers of events potentially undermine the feasibility of analyses focusing on risk of late SPCs, after stratification according to specific region of origin. This said, our explanation is only speculative.

Third, cumulative incidence rates at 10 years after PCa diagnosis for RP and RT vs. RP were 12.0 vs. $8.7 \%, 2.0$ vs. $1.2 \%, 2.1$ vs. $1.3 \%$ and 9.9 vs. $7.4 \%$ for overall SPCs, primary pelvic SPCs, overall pelvic SPCs and non-pelvic SPCs, respectively. These findings 
demonstrate that RP and RT patients have higher incidence of SPCs than RP alone patients. Moreover, it is noteworthy that one out of ten PCa patients will develop a SPC at 10 years of follow-up. Therefore, the follow-up of RP patients, especially those treated with a combination of RT, should not only focus on identification of biochemical recurrence but also on detection of SPCs. Depending on the characteristics of the population, the rate of SPCs may even approach the rate of BCR. This consideration should be given equal importance within the urologic, as well as radiation oncology communities, so that most effective SPC detection strategies are employed.

Fourth, in multivariable Cox regression models RP and RT represented an independent predictor for overall SPCs, primary pelvic SPCs, overall pelvic SPCs, non-pelvic SPCs, late overall SPCs and late non-pelvic, compared to RP. However, RP and RT was not associated with an increased risk for late primary pelvic and late overall pelvic SPCs. This results differ from those reported by Abdel-Wahab et al., who found that radiation after radical surgery increased late primary pelvic SPCs but not non-pelvic SPCs. ${ }^{8}$ However, their report was based on a historic population (1973-2002) and changes in the radiotherapy could have led to the different results. Moreover, their report was based on a smaller group of RP and RT treated patients $(n=5,044)$ compared to the current study $(n=10,870)$.

Taken together, our analyses suggest that RT results in a higher risk of SPCs, compared to patients treated with RP. Therefore, careful patient surveillance and detection efforts aimed at identifying SPCs after combined RP and RT are of crucial importance. In that regard, we are unaware of specific follow-up protocols. In consequence, SPCs may be missed in patients, who have a favorable cancer control profile and are no longer closely followed. In consequence, urology and/or radiation oncology follow-up should continue well beyond 10 years after RP and RT delivery, to minimize or ideally eliminate the probability of missing an SPC. However, despite the higher risk of SPCs after RT and RP, it is important also to weigh the potential curative benefit of salvage or adjuvant RT. This always should be taken into account in the risk-benefit discussion with our patients when RT is considered. Moreover, our data clearly show that the absolute rate of SPC after RP and RT did not increase considerably. This said, it is still a matter of fact that the screening of all patients treated with RP and RT cannot be recommended or supported based on our data. Last but not least, no increased risk for late primary pelvic SPCs was recorded. Therefore, further studies, ideally with even longer follow-up, are welcome to confirm these findings.

Some important limitations need to be acknowledged. First and foremost, it shares limitations of all similar studies that were based on the SEER database and relied on retrospective data with a high potential for bias due to selection of PCa patients. Additionally, information on radiation dose and modus are unavailable in the SEER database, which might have changed during the study span and could have influenced our results. Furthermore, the maturity of our database prevented us from addressing SPC incidences beyond 10 years due to insufficient numbers of patients at risk and excessively small numbers of events. Moreover, it is possible that important differences persisted, according to variables that are unavailable in retrospective databases, such as the SEER database. Such variables include, baseline performance status (Eastern Cooperative Oncology Group), smoking status and comorbidities. Moreover, the SEER database lacks of information about ADT. In 
consequence, despite the most stringent statistical adjustment for potential differences, our findings may be undermined by residual biases. Their presence and magnitude of their potential effects will only be known once prospective randomized trials address the same endpoint that we have addressed in the current analysis. Unfortunately, to the best of our knowledge, no such trials are underway or have been planned. In consequence, we hope that our findings may add to the existing knowledge and further retrospective studies will aim to validate our results. Finally, despite the large sample size, the current study cohort only represents a $30 \%$ population sample with inherent limitations. ${ }^{13}$

In conclusion, RP with RT was associated with moderately increased risk of SPCs, compared to RP alone. This observation should be thoroughly discussed at informed consent and considered during follow-up. 


\section{References}

1. Bolla M, van Poppel H, Collette L, et al. Postoperative radiotherapy after radical prostatectomy: a randomised controlled trial (EORTC trial 22911). Lancet. 2005;366(9485):572-578.

2. Da Pozzo LF, Cozzarini C, Briganti A, et al. Long-term follow-up of patients with prostate cancer and nodal metastases treated by pelvic lymphadenectomy and radical prostatectomy: the positive impact of adjuvant radiotherapy. Eur Urol. 2009;55(5):1003-1011.

3. Abdollah F, Suardi N, Cozzarini C, et al. Selecting the optimal candidate for adjuvant radiotherapy after radical prostatectomy for prostate cancer: a long-term survival analysis. Eur Urol. 2013;63(6):998-1008.

4. Thompson IM, Jr., Tangen CM, Paradelo J, et al. Adjuvant radiotherapy for pathologically advanced prostate cancer: a randomized clinical trial. JAMA. 2006;296(19):2329-2335.

5. Mahmood S, Vu K, Tai P, et al. Radiation-induced second malignancies. Anticancer Res. 2015;35(4):2431-2434.

6. Jin T, Song T, Deng S, Wang K. Radiation-induced secondary malignancy in prostate cancer: a systematic review and meta-analysis. Urol Int. 2014;93(3):279-288.

7. Davis EJ, Beebe-Dimmer JL, Yee CL, Cooney KA. Risk of second primary tumors in men diagnosed with prostate cancer: a population-based cohort study. Cancer. 2014;120(17):2735-2741.

8. Abdel-Wahab M, Reis IM, Wu J, Duncan R. Second primary cancer risk of radiation therapy after radical prostatectomy for prostate cancer: an analysis of SEER data. Urology. 2009;74(4):866-871.

9. Zaorsky NG, Harrison AS, Trabulsi EJ, et al. Evolution of advanced technologies in prostate cancer radiotherapy. Nat Rev Urol. 2013;10(10):565-579.

10. Abdel-Wahab M, Reis IM, Hamilton K. Second primary cancer after radiotherapy for prostate cancer--a seer analysis of brachytherapy versus external beam radiotherapy. Int J Radiat Oncol Biol Phys. 2008;72(1):58-68.

11. Preisser F, Bandini M, Mazzone E, et al. Validation of the Social Security Administration Life Tables (2004-2014) in Localized Prostate Cancer Patients within the Surveillance, Epidemiology, and End Results database. Eur Urol Focus. 2018.

12. D'Amico AV, Whittington R, Malkowicz SB, et al. Biochemical outcome after radical prostatectomy or external beam radiation therapy for patients with clinically localized prostate carcinoma in the prostate specific antigen era. Cancer. 2002;95(2):281-286.

13. Marchioni M, Bandini M, Pompe RS, et al. Survival of metastatic renal cell carcinoma patients continues to improve over time, even in targeted therapy era. International urology and nephrology. 2017. 


\section{Figures and Tables}

Fig. 1. Cumulative incidence plots depicting incidence of overall secondary primary cancers (SPC), pelvic SPC, primary pelvic SPC, non-pelvic SPC for radical prostatectomy and radiotherapy vs. radical prostatectomy alone treated patients. $\mathrm{PCa}$ : prostate cancer.

Figure 1
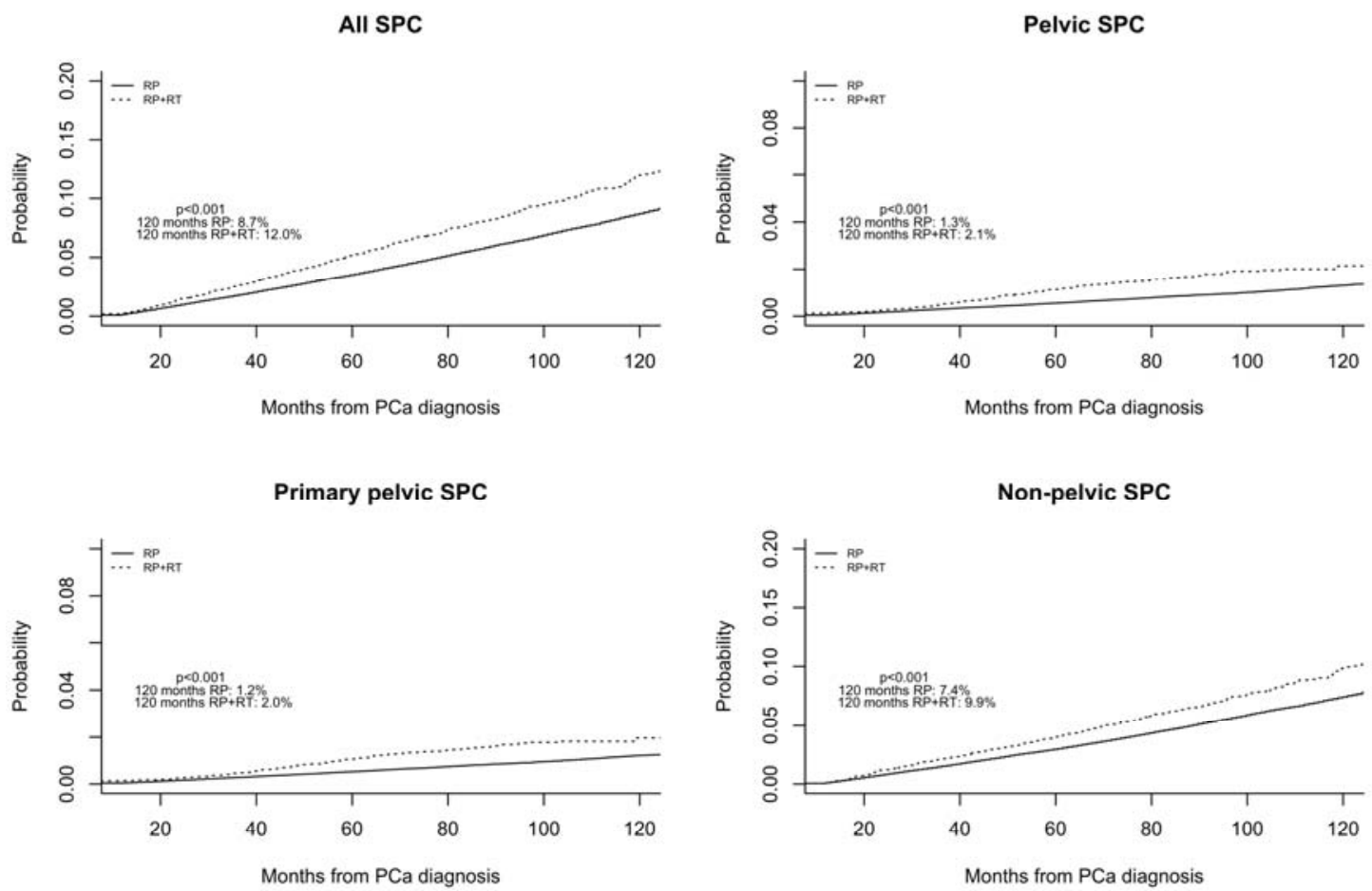

Table 1. Descriptive characteristics of patients diagnosed with prostate cancer and treated with radical prostatectomy and radiotherapy or radical prostatectomy alone, within the SEER database between 2004 and 2015

\begin{tabular}{|c|c|c|c|c|}
\hline & $\begin{array}{c}\text { Overall } \\
(n=152161)\end{array}$ & $\begin{array}{c}\text { RP+RT } \\
(n=10870,7.1 \%)\end{array}$ & $\begin{array}{c}\text { RP } \\
(n=141291, \\
92.9 \%)\end{array}$ & $\mathbf{p}$ \\
\hline $\begin{array}{l}\text { Year of prostate cancer } \\
\text { diagnosis, median } \\
\text { (interquartile range) }\end{array}$ & $\begin{array}{c}2009 \\
(2007-2011)\end{array}$ & $\begin{array}{c}2009 \\
(2006-2011)\end{array}$ & $\begin{array}{c}2009 \\
(2007-2011)\end{array}$ & 0.008 \\
\hline $\begin{array}{l}\text { Age at prostate cancer } \\
\text { diagnosis (years), } \\
\text { median } \\
\text { (interquartile range) }\end{array}$ & $\begin{array}{c}61 \\
(56-66)\end{array}$ & $\begin{array}{c}63 \\
(57-69)\end{array}$ & $\begin{array}{c}61 \\
(56-66)\end{array}$ & $<0.001$ \\
\hline $\begin{array}{l}\text { PSA (ng/ml), median } \\
\text { (interquartile range) }\end{array}$ & $\begin{array}{c}5.7 \\
(4.4-8.2)\end{array}$ & $\begin{array}{c}7.9 \\
(5.2-14.5)\end{array}$ & $\begin{array}{c}5.6 \\
(4.4-8.0)\end{array}$ & $<0.001$ \\
\hline D'Amico risk, n (\%) & & & & \\
\hline
\end{tabular}




\begin{tabular}{|c|c|c|c|c|}
\hline Low-risk & $\begin{array}{l}37777 \\
(24.8) \\
\end{array}$ & $\begin{array}{l}984 \\
(9.1) \\
\end{array}$ & $\begin{array}{l}36793 \\
(26.0) \\
\end{array}$ & $<0.001$ \\
\hline Intermediate- risk & $\begin{array}{l}66046 \\
(43.4)\end{array}$ & $\begin{array}{l}3732 \\
(34.3)\end{array}$ & $\begin{array}{l}62314 \\
(44.1)\end{array}$ & \\
\hline High-risk & $\begin{array}{l}33910 \\
(22.3)\end{array}$ & $\begin{array}{c}5626 \\
(51.8)\end{array}$ & $\begin{array}{l}28284 \\
(20.1)\end{array}$ & \\
\hline NA & $\begin{array}{c}14428 \\
(9.5)\end{array}$ & $\begin{array}{c}528 \\
(4.8) \\
\end{array}$ & $\begin{array}{c}13900 \\
(9.8)\end{array}$ & \\
\hline \multicolumn{5}{|l|}{$\begin{array}{l}\text { Clinical tumor stage, } n \\
(\%)\end{array}$} \\
\hline$<\mathrm{T} 2$ & $\begin{array}{l}97565 \\
(64.2)\end{array}$ & $\begin{array}{c}6013 \\
(55.3) \\
\end{array}$ & $\begin{array}{l}91552 \\
(64.8)\end{array}$ & $<0.001$ \\
\hline $\mathrm{T} 2$ & $\begin{array}{l}50876 \\
(33.4) \\
\end{array}$ & $\begin{array}{r}3789 \\
(34.9) \\
\end{array}$ & $\begin{array}{l}47087 \\
(33.3) \\
\end{array}$ & \\
\hline$\geq \mathrm{T} 3$ & $\begin{array}{l}3720 \\
(2.4)\end{array}$ & $\begin{array}{l}1068 \\
(9.8) \\
\end{array}$ & $\begin{array}{l}2652 \\
(1.9)\end{array}$ & \\
\hline \multicolumn{5}{|l|}{ Biopsy GGG, n (\%) } \\
\hline 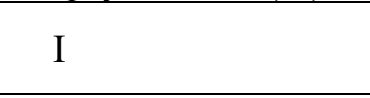 & $\begin{array}{l}56505 \\
(37.1) \\
\end{array}$ & $\begin{array}{c}1716 \\
(15.8) \\
\end{array}$ & $\begin{array}{l}54789 \\
(38.8) \\
\end{array}$ & $<0.001$ \\
\hline II & $\begin{array}{l}51977 \\
(34.2) \\
\end{array}$ & $\begin{array}{l}2755 \\
(25.4)\end{array}$ & $\begin{array}{l}49222 \\
(34.8)\end{array}$ & \\
\hline III & $\begin{array}{l}17797 \\
(11.7) \\
\end{array}$ & $\begin{array}{c}1863 \\
(17.1) \\
\end{array}$ & $\begin{array}{l}15934 \\
(11.3) \\
\end{array}$ & \\
\hline IV & $\begin{array}{c}10306 \\
(6.8)\end{array}$ & $\begin{array}{l}1719 \\
(15.8)\end{array}$ & $\begin{array}{l}8587 \\
(6.1) \\
\end{array}$ & \\
\hline $\mathrm{V}$ & $\begin{array}{l}7315 \\
(4.8)\end{array}$ & $\begin{array}{l}2129 \\
(19.6) \\
\end{array}$ & $\begin{array}{l}5186 \\
(3.7)\end{array}$ & \\
\hline NA & $\begin{array}{l}8261 \\
(5.4)\end{array}$ & $\begin{array}{c}688 \\
(6.3) \\
\end{array}$ & $\begin{array}{l}7573 \\
(5.3)\end{array}$ & \\
\hline
\end{tabular}

GGG: Gleason grade group; N: not available; PSA: prostatic-specific antigen value; RP: radical prostatectomy; RT: radiotherapy; SEER: Surveillance, Epidemiology, and End Results.

\begin{tabular}{|c|c|c|c|c|}
\hline & $\begin{array}{c}\text { Overall } \\
(n=152161)\end{array}$ & $\begin{array}{c}\text { RP+RT } \\
(n=10870)\end{array}$ & $\begin{array}{c}\text { RP } \\
(n=141291)\end{array}$ & $\mathbf{p}$ \\
\hline No SPC, n (\%) & $144306(94.8)$ & $10150(93.4)$ & $134156(95)$ & $<0.001$ \\
\hline Overall SPCs, $\mathrm{n}(\%)$ & $7855(5.2)$ & $720(6.6)$ & $7135(5.0)$ & \\
\hline $\begin{array}{l}\text { Median time in month to SPC } \\
\text { (interquartile range) }\end{array}$ & $\begin{array}{c}51.4 \\
(29.2-78.6) \\
\end{array}$ & $\begin{array}{c}48.4 \\
(28.2-73.6) \\
\end{array}$ & $\begin{array}{c}51.4 \\
(29.2-78.6) \\
\end{array}$ & 0.04 \\
\hline Overall SPCs, n (\%) & $7855(5.2)$ & $720(6.6)$ & $7135(5)$ & $<0.001$ \\
\hline Pelvic SPCs, $\mathrm{n}(\%)$ & $1228(0.8)$ & $140(1.3)$ & $1088(0.8)$ & $<0.001$ \\
\hline Primary pelvic SPCs, n (\%) & $1137(0.7)$ & $130(1.2)$ & $1007(0.7)$ & $<0.001$ \\
\hline Non-pelvic SPCs, n (\%) & $6627(4.4)$ & $580(5.3)$ & $6047(4.3)$ & $<0.001$ \\
\hline Late overall SPCs ( $>5$ years), $n$ & $3323(2.2)$ & $279(2.6)$ & $3044(2.2)$ & 0.005 \\
\hline
\end{tabular}




\begin{tabular}{|l|c|c|c|c|}
\hline$(\%)$ & & & & \\
\hline Late-pelvic SPCs, $\mathrm{n}(\%)$ & $474(0.3)$ & $43(0.4)$ & $431(0.3)$ & 0.1 \\
\hline $\begin{array}{l}\text { Late primary pelvic SPCs, } \mathrm{n} \\
(\%)\end{array}$ & $431(0.3)$ & $40(0.4)$ & $391(0.3)$ & 0.1 \\
\hline Late non-pelvic SPCs, $\mathrm{n}(\%)$ & $2849(1.9)$ & $236(2.2)$ & $2613(1.8)$ & 0.02 \\
\hline $\begin{array}{l}\text { SPCs stratified according to } \\
\text { sites, } \mathrm{n}(\%)\end{array}$ & & & & \\
\hline Anus & $17(0)$ & $1(0)$ & $16(0)$ & $<0.01$ \\
\hline Bladder & $901(0.6)$ & $109(1)$ & $792(0.6)$ & \\
\hline Blood \& bone marrow & $585(0.4)$ & $56(0.5)$ & $529(0.4)$ & \\
\hline Colon & $586(0.4)$ & $46(0.4)$ & $540(0.4)$ & \\
\hline Esophagus & $161(0.1)$ & $20(0.2)$ & $141(0.1)$ & \\
\hline Kidney \& renal pelvis & $639(0.4)$ & $40(0.4)$ & $599(0.4)$ & \\
\hline Liver & $146(0.1)$ & $18(0.2)$ & $128(0.1)$ & \\
\hline Lung \& pleura & $1236(0.8)$ & $134(1.2)$ & $1102(0.8)$ & \\
\hline Others & $1780(1.2)$ & $152(1.4)$ & $1628(1.2)$ & \\
\hline Pancreas & $384(0.3)$ & $38(0.3)$ & $346(0.2)$ & \\
\hline Penile & $6(0)$ & $1(0)$ & $5(0)$ & \\
\hline Recto-sigmoid & $56(0)$ & $6(0.1)$ & $50(0)$ & \\
\hline Rectum & $186(0.1)$ & $15(0.1)$ & $171(0.1)$ & \\
\hline Skin & $914(0.6)$ & $63(0.6)$ & $851(0.6)$ & \\
\hline Stomach & $223(0.1)$ & $17(0.2)$ & $206(0.1)$ & \\
\hline Testis & $21(0)$ & $2(0)$ & $19(0)$ & \\
\hline Urethra & $3(0)$ & $0(0)$ & $3(0)$ & \\
\hline Ureter & $11(0)$ & $2(0)$ & $9(0)$ & \\
\hline RP radicaprostan & & & \\
\hline
\end{tabular}

RP: radical prostatectomy; RT: radiotherapy; SPC: secondary primary cancer; SEER:

Surveillance, Epidemiology, and End Results. 


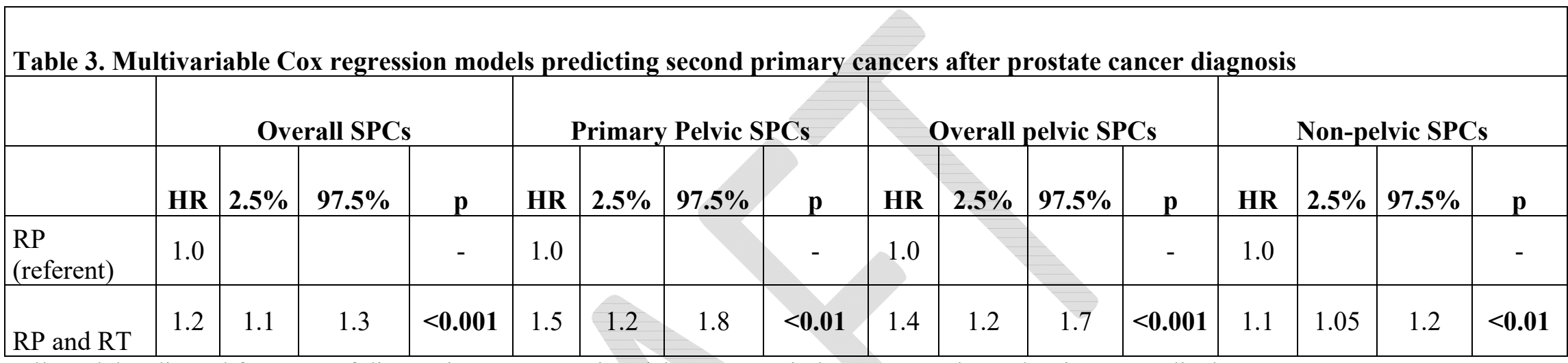

All models adjusted for: year of diagnosis, age, D’Amico risk, race, marital status. HR: hazard ratio; RP: radical prostatectomy; RT: radiotherapy; SPCs: secondary primary cancers.

\begin{tabular}{|c|c|c|c|c|c|c|c|c|c|c|c|c|c|c|c|c|}
\hline & \multicolumn{4}{|c|}{ Overall SPCs } & \multicolumn{4}{|c|}{ Primary pelvic SPCs } & \multicolumn{4}{|c|}{ Overall pelvic SPCs } & \multicolumn{4}{|c|}{ Non-pelvic SPCs } \\
\hline & HR & $2.5 \%$ & $97.5 \%$ & $\mathbf{p}$ & HR & $2.5 \%$ & $97.5 \%$ & $\mathbf{p}$ & HR & $2.5 \%$ & $97.5 \%$ & $\mathbf{p}$ & HR & $2.5 \%$ & $97.5 \%$ & $\mathbf{p}$ \\
\hline RP (referent) & 1.0 & & 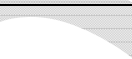 & - & 1.0 & +2 & +2 & - & 1.0 & & & - & 1.0 & & & - \\
\hline $\mathrm{RP}$ and RT & 1.2 & 1.03 & 1.3 & 0.01 & 1.3 & 0.9 & 1.8 & 0.2 & 1.2 & 0.9 & 1.7 & 0.2 & 1.2 & 1.01 & 1.3 & 0.03 \\
\hline
\end{tabular}

All models adjusted for: year of diagnosis, age, D'Amico risk, race, marital status. HR: hazard ratio; RP: radical prostatectomy; RT: radiotherapy; SPCs: secondary primary cancers. 